\title{
Author Index, Vol. 77, No. 1-2, 1997
}

Abazis,D. 86, 112 Abramchik, E. 97 Acar,A. 100, 106, 140 Acar, G. 115 Acar,H. 100,106, 140 Acar,O. 140 Adamov, M.M. 75 Adamski, E.L. 27 Addor,M.-C. 101 Adnet,J.J. 56 Adouard,V. 101 Adzic, S. 85 Aguilar, J.LI. 135 Ajzemberg, M. Ill Akopian, G. 32 Akopian, G.R. 127 Akta§,D. 86,87,140 Alard,J.P. 74 Alberti,C. 55 Albrechts, J.C.M. 65 Albuisson, E. 32 Alegre, M. 94 Aleinikova, O. 135 Aleksandrovic, S. 143 Alembik,Y. 116 Alembik,Y. 86,123 Alexandrov, LA. 67 Alexiou,N. 121 Alikasifoglu, M. 86 Alkalm,N. 140 Alvarez, S. 84 Alves,C. 128 Ambrósio,P. 93, 128 Amiel, A. 127 Ammini,A.C. Ill,113 Amorim, A. 119 Anandakumar, C. 90 Anastasiadou, V. 101 Andersen, C.L. 53 Andreeva, TE. 38 Andreozzi, L. 39 Andréo,B. 53,62 Angelakis, Ph. 31 Angevin, E. 144 Anichini, A. 75 Antonacci, R. 158 Antonelli,A. 105 Antonenko, V.G. 101 Antsaklis, A. 94 Antunovic, P. 130 Apak,M.Y. 58,92 Aphanasieva, N.A. 154 Archidiacono, N. 34 Archimbaud, E. 130 Ardanaz,M.T. 129 Ardisia,C. 86 Argusti, A. 43 Armengol, G. 140 Arnold, J. 74 Arranz,E. 128, 138 Arrieta, I. 102 Arrigo, G. 40 Arruga, M.V. 39

Arsic, N. 67 Artan, S. 88, 102, 150 Arveiler, B. 124 Asko-Seljavaara, S. 140 Aslanis,P. 80 Aspinwall, P. 28 Atanasova, S. 110 Atici,A. 124 Atkey,N.W. 85 Audibert, F. 87 Aurias,A. 104, 147 Ausio, A. 151 Ausserer, B. 103 Autio,S. 124 Avigad, S. 52 Aviram-Goldring, A. 48, 55 Avivi,L. 127 Avramopoulos, D. 79 Ayoutantis, M. 138 Aytan,M. 58 Azemar, V. 56 Azzarelli, A. 52

Babunashvili, M. 90 Badia,J. 87 Baena,N. 87 Bahçe,M. 75,117, 136 Bahge,M. 95 Bajnoczky, K. 79 Bakiri,M. 139 Balashova, E.V. 76 Balci,S. 87, 140 Ballarin,J. 73 Ballesta,F. 89, 115 Balogh,E. 141 Balzani, O. 77 Bar-Am, I. 24,52, 133 Baranov, A. 37 Baranov, V. 32 Baranov, V.S. 33,87,96 Baranova, H. 32 Barbaric, I. 72 Barbêdo,C. 120 Barbet,P. 113 Bardi,A. 128 Bardi,G. 144 Bardoni, B. 40 Barilyak, I. 68, 84 Barilyak, I.R. 154 Barkai, G. 48, 55 Barnicoat, A.J. 40 Barnoux, M. Ill Barrera,J.C. 58,96,109 Barrett, I.J. 80 Barri,P.N. 77 Barrios, L. 31, 57 Bartelings, M. 88 Bartsch, O. 63 Bartsev,D.V. 76 Bartseva, O.B. 76, 156 Baryliak,I. 65, 157 Bârzu,M. 143 Basaran,A. 102 Ba§aran,N. 88, 102, 118, 150 Basaran, S. 58,92 Bassi,L. 72,141 Batanian, J. 127 Baty,D. 107 Bau, A. 94 Baur,A.S. 51 Bay,J.O. 32 Bayol,Ü. 107 Baysal,C. 114 Bazin,A. 87,97 Beà,S. 147 Bechet, D. 74 Bedelbaeva, K.A. 56,76,

101 Begovic, D. 60 Beksaç, S. 87 Belengeanu, V. 125 Belhabri,A. 130 Bellec,V. 43,58,76,83, 84,85 Ben Ahmed, M. 58 Benet,J. 77,82 Benevento, A. 131 Benga,Gh. 74 Benitez,J. 138 Benizio, S. 77, 88 Benkhalifa, M. 43,58,76, 82,83,85,112 Benner, A. 25 Bensimon, A. 28, 33 Benítez, J. 128 Benzacken,B. 57,76,84 Berdina,L.M. 126,154 Bereshova, A.K. 79 Bergendi, E. 120 Berger, R. 46 Bergere,M. 78 Bergère, M. 76,84 Berishvüi, M. 90 Berker Karaüzüm, S. 115 Berker-Karaüzüm, S. 141 Bernardi, G. 22, 39 Bernasconi, B. 51, 149 Bernheim, A. 54, 144 Bernstein, J. 28 Bernués, M. 141, 142 Berry, T.L. Ill Bertani, E. 143 Bertheas, M.F. 101 Beryozkina, E.V. 70, 144, 155 Beslid,N. 104 Besses, C. 131 Bettio,D. 142 Betts, D.R. 142 Bever, Y. van 115 Beverstock, G.C. 88 Bialecka,M. 128 Bieger,W.P. 29 
Bielińska, B. 110 Bignon,Y.J. 32 Bigoni,R. 128 Binkert,F. 102,103 Biondi,A. 139 Birnbacher, R. 59 Bishop, M. 151 Bissig,H. 147 Blanchet,P. 114 Btaszczyk, A. 70 Blennow,E. 65, 102,123 Blinnikova, O.E. 29,76,

127 Blomqvist, C. 140 Bloomfield, CD. 136 Boada,M. 77 Boaventura 96, 119 Boavida,M.G. $89,93,103$,

128 Boban,D. 136 Bocca,P. 146 Bocian, E. 53 Boduroglu, K. 87 Bogdanic, V. 136

Bogomasova, A. 31 Böhling,T. 140 Bøhrk,J. 144 Boieiro, F. 93,103 Bolund,L. 54 Bomme, L. 144 Bondeux, N. 60 Bonduelle,M. 100 Bongso, A. 90 Bonuccelli, U. 116 Borgaonkar, D.S. 34 Bornfleth,H. 23 Borrell,A. 89 Borroni,C. 89 Bosma, A.A. 37 Boström, J. 52 Bot,F. 65 Botas,M. 108 Bothorichvilli, R. 32 Boumazeau, J.A. 46 Bourgeois, C.A. 82 Bourgeois, J.M. 89 Boyd,E. 45,117 Bradl,J. 23

Brajenovic-Milic, B. 72, 95 Brambilla, E. 61 Brandt, C.A. 54 Brankovic, S. 67 Brecevic,L. 102,103,142 Brem,G. 152 Brenner, B. 50 Bresson, J.-L. 103,107 Bresson, J.L. 81 Breuning, M. 124 Brewer, C. 117 Brezinová, J. 135 Briançon, G. 134 Briault, S. 91 Brieger, J. 50

Brisdelli, F. 105 Brownie, J. 34 Bruhat,M. 32 Brühl,P. 28 Brunner,R.M. 37 Buchinskaya, L.G. 142 Bukvic,N. 103 Bulgheroni, C. 99 Bull,J.H. 34 Bureau, J.P. 89 Bureo,E. 138 BureticTomljanovic, A.

68 Burkin,D.J. 26,61 Burridge, M. 138 Bustos, J.E. 55 Buteica, E. 121 Butler, T.A. 134 Butomo,I.V. 80,92 Buzhievskaya, T.I. 77 Bychkova, A. 115 Bychkova, A.M. 154

Caballin, M.R. 87 Caballín, M.R. 140,141,

142 Cabello,P. 129,131 Cacciò,S. 39 Cacheux, V. 60 Caferler,J. 88 Calabrese, G. 28 Calasanz, M.J. 129,138 Calderone, C. 52 Calender, A. 114 Cali, A. 62 Caligiani, R. 75 Callens,B. 66 Calò,A. 88 Camandona, F. 77 Camerino, G. 40 Campagna, V. 99 Campbell, L.J. 130 Campbell, R.D. 54 Campo, E. 135 Canapa, A. 153 Candelier, J.J. 53, 54 Canis, M. 32 Canki-Klain, N. 104 Cankus,G. 136 Cankus, G. 75 Cantu,J.M. 42 Capella,C. 51,149 Capodiferro, F. 28 Cappellini, A. 89 Capriglione, T. 37 Caputo,V. 153 Cararach,J. 89 Carbonell,E. 30 Cardy,D.L.N. 26 Carlucci, S. 77,88 Carrera, M. 77 Carrió,A. 89, 115 Carroll, A.J. 136

\section{KÄIUiEH}

E-Mail karger@karger.ch Fax+4 41613061234 http://www.karger.ch

(C) 1997 S. Karger AG, Basel

159

Carvalhas,R. 104 Carvalho,B. 119 Carvalho,L. 108 Casadevall, C. 141, 142 Casalaro, A. 146 Casalone, R. 143 Castagné, C. 129 Castedo,S. 104, 105, 119 Castoldi, G.L. 128 Castorina, P. 52 Cavalli, P. 88 Cavani, S. 43,77 Cazzaniga, G. 139 Celeda,D. 29 Cerati,M. 143 Cerbah,M. 54 Cerveró,C. 131 Ceylaner, S. 106 Chaabouni, H. 129 Chaplin, T. 47 Charbonnier, J.F. 104 Chardon,P. 38 Charlieu, J.P. 62 Charrin, C. 130 Chassagne, J. 74 Chatelain,C. 104 Chaussain, J.L. 113 Chaves,J. 104 Chaze,A.M. 114 Cheadle,J. 28 Chelo,E. 75 Chen,H. 63 Chesnokova, G.G. 29, 117,

127 Chevret, E. 54 Chéchile, G. 141 Chiesa,J. 89 Chita, O. 121 Chmielińska, E. 119 Chrissikopoulos, A. 98 Christopoulou, S. 110 Chrzanowska, K. 128 Chrzanowska, K.H. 41 Chudoba,D. 93 Chudoba, I. 32, 59, 120 Ciccarese, S. 158 Cifuentes, P. 77, 82 Cigudosa, J.C. 129 Çilingir,O. 118 Clark, R. 35,134 Claussen, U. 32, 36 Clouston,HJ. 49 Coggi,G. 131 Cohen, I. 52 Colak,A. 118 Çolak,A. 119 Colenbrander, B. 37 Coll,M.D. 109 Collot,N. 60 Colls, P. 78 , 85 Colombo, D. 89 Colombo, L. 149 Colovic,M. 130 Colovic,M. 137 Connor, J.M. 45 Cook, Y. 130 Copin,H. Ill Coprean,D. 143 Çora,T. 100 Cordeiro,I. 104, 105 Corona, M. 87 
Correia, H. 104 Correia,H.O. 89 Correira,H. 93 Corrias,M.V. 146 Costa, D. 115 Costa, D. 89 Costalos, C. 121 Coulaud, J. 54 Coullin,P. 38,54 Coullin,Ph. 53 Covic, M. 116 Covic, M. 94 Craig,J. 56 Craig,J.M. 27,55 Craven, MJ. 43,44 Cremer, C. 23, 56, 64 Cremer, T. 23, 25,27,51, 55,56,58 Creus,A. 30 Criado,B. 104,105 Cîrlan,M. 150 Croce,C.M. 50 Crolla,J.A. 56 CruzCigudosa, J. 138 Cücer,N. 106 Culic,S. 105 Culic,V. 105 Cuneo,A. 128 Curotti, G. 83 Cusidó, L. 109 Cusido,L. 58 Cusidó,L. 96 Czarnecka, M. 131

Dabrowski, P. 148 Dada,R. 78 Dadoune,J.P. 82,84 Dagna Bricarelli, F. 43, 62, 77 Dakhno,F. 84 Dalakishvili, M. 90 D’Alessandro, E. 105 Dalprà,L. 52,89, 126, 145,147 Damatopoulou, A. 31 Danesino, C. 137 Daniely,M. 48,55 Dardoufas, K. 31 Da Suva, F. 60 Daumer Haas, C. 105 Davarashvili, D. 90 Davelaar, I. 65 Davidson, N. 117 Davison,E.V. 90,98 Davriendt, K. 40 Dean,N. 130 De Bernardo, C. 106 Debevec,M. 83,104 DeBiasio,P. 97 DeCatte, L. 100 Decker,HJ. 50 DeCoteau, J.F. 133 DeGeeter,B. 116 Dehaese,O. 104 Delhanty, J.D.A. 49 Dellavecchia, C. 137 Delon,B. 58 Del Pino, O. 113 Del Porto, G. 106,109 Demaille,J. 114 Demarcq, C. 61

Demertzi, I. 92 Deméocq, F. 134 Demidova, I.A. 66,79, 106, 126 Demina,N $\Lambda$. 29,117,127 Demircan, A. 141 Demirhan, O. 124 Demirtas, H. 106 Demirtas, H. 68,69 Depetris, D. 64 De Polanco, M.M.E. 55 Desmaze, C. 55 DeSmedt, D. 100 Dev,V.G. 100 Devriendt, K. 24, 108 DeWitt,M.I. Ill Dhanjal,S. 45 Dhermain, J. 74 Diadic, T.G. 76 Dibskiy,S.S. 73 Dierlamm, J. 46, 57 Dietzel, S. 23 Diez,J.L. 138 DiMeo, G.P. 151 Dimitriou, E. 137 Dineen,T.M. 27,79 Dionet,C. 74 DiRenzo, G. 86 Dirks, R. 65 DiRosa,C. 106 Djordjevic,V. 130 Djordjevic, V.A. 62 Dockery, H.E. 43,44 Doco-Fenzy, M. 56 Dominguez, M.C. 109 Dommergues, M. 87 Donmez,H. 68,69 Donnarieix, D. 74 Donti,E. 86 Dor,J. 48 Dordevic, V. 137 Dott,B. 86,123 Doud,L. 151 Douglas, A. 130 Drobnic,S. 83 Drosdovskaya, N.A. 97 Dubovic,B.V. 72 Duchesne, V. 83 Dullens,H. 148 Dumanski, J.P. 50 Dumitrescu, R. 74 Dunlop,A. 117 Dupuy,O. 124 Durães,I. 96,119, 120 Durak,B. 88,102,118,

150 Durak,T. 75,117 Durakbasi, H.G. 106 Duran,C. 87 Duran,E.H. 117 Duran,Eh. 95 Durand,V. 64,73 Durisic,M. 91, 143 Durm,M. 56,64 Durst, M. 64 Dursun,N. 68 Dussaulx, E. 53 Dutly,F. 102,103 Dutrillaux, B. 25,82 Dysheva, N. 75 Dzubenko, M. 65, 157

Earle, E. 66 Efimova,G.N. 75 Egenter,C. 51,62, 147 Eggen, A. 151 Egozcue, J. 38, 48, 73, 77 , $78,81,82,85,94,141$,

142, 147, 153 Eils,R. 23 Ejarque, M.J. 141 Ekman, P. 51 Ekong,R. 28

Ekshiyan,A. 154, 156, 157 Elbistan, M. 118 Elezovic, I. 137 Elloumi, H. 129 El Mouatassim, S. 143 Elomaa,I. 140 Engelen, J.J.M. 65 Engels,H. 107 English, C.J. 85 Enseñat, C. 153 Epicoco, G. 86 Erbengi, A. 140 Erçal,D. 87,107 Erdem,G. 117 Erdogan, A. 141 Erenmemisoglu, A. 69 Erkul,í. 100 Ernst, S. 81 Escudero, A. 128 Escudero, T. 109 Escudier, B. 144 Espinet,B. 131, 135,138 Essed,C. 148 Esteve, J. 135 Estivill,X. 115 Estivill,X. 89 Evans, J. 85 Evgrafov, O.V. 56 Evteev,A.V. 38, 153 Ezquieta, B. 155, 157

Fabbri,A. 51,149 Faber,H.H. 56,65 Facco,C. 51,99 Faed,M.J.W. 107 Falcinelli, C. 90 Falzetti,D. 57 Farmer, M. 35 Fasce, V. 45,100 Fasola,M. 99 Fassotte, M.F. 132 Faugeras, Y. 74, 143 Faure,J.M. 89 Fedele, A. 77, 88 Federico, C. 39 Fedorov,S.N. 145 Fedortseva, R. 154 Fedortseva, R.F. 69, 71 Feijoo, M.J. 108 Feingold,J. 124 Fejgin,M. 127 Fellmann,F. 103, 107 Fellous, M. 113 Feng, J. 51 Fenger, C. 144 Fenwick, J. 49 Ferguson-Smith, M.A. 24, 26,27,39,61,63,152 Fernandez, R. 78 Ferraz, F. 103

Ferreira Pinto, C. 108 Ferreres, J.C. 87 Ferretti, L. 151 Ferro,M.T. 30, 129, 131 Fews, G.A. 90,98 Fillet, G. 132 Fisher, G. 84 Fletcher, J. A. 133 Flint, J. 26 Florensa, L. 131 Fogu, G. 137 Forabosco, A. 43, 90 Fortuna,A.M. 104, 105 Fortuny, A. 89 Forus, A. 140 Fougerousse, F. 28 
Foxley,R.A. Ill Fratello,A. 34 Fraticelli, E. 62 Freesemann, S.M. Ill Fréminville, B. de 101 Friedrich, U. 54 Frusconi, S. 75 Frydman, N. 78 Fryns, J.-P. 24, 108 Fryns, J.P. 40 Fryns, J.-P. 40 Fryns, J.P. 42 Fryssira,H. 108, 112 Fucic, A. 69 Fuhrman Conti, A.M. 52, 145,147 Furlan,D. 51,99 Furtado,J.M. 89,93 Fuster, C. 73, 94, 147

Gabau,E. 87, 109 Gaber,E. 127 Gaginskaya, E.R. 38 Gagos, S. 57 Gaide,A.-C. 101 Galla,A. 65,122,123 Gallambrun, C. 101 Galofrel,P. 30 Gambarelli, D. 149 Gancedo,P.G. 155,157 Gandolfi,P. 95 Ganina,K,P. 142 Gänshirt, D. 45 Garagna,S. 90 Garaj-Vrhovac, V. 69 GarcíaSagredo, J.M. 30,

36,131 Garcia, F. 38 Garcia, L. 138 Garcia, M. 38,81 Garcia-Sagredo, J.M. 42 Garcia Santiago, F. 129 Garini, Y. 24 Garritsen, H.S.P. 45 Gasenko, E. 84 Gasser,T.C. 51 Gatta,V. 28 Gauthier, J.P. 143 Gelabert,A. 141, 142 Geneix,A. 108,122 Gentile, M. 103 Georghiou, A. 27, 64 Georgieva, V. 71 Georgiou, I. 101 Geraudel, A. 116

160

Cytogenet Cell Genet Vol. 77, No. 1-2, 1997

Gerdes,A.-M. 144 Gerdes,T. 60 Gerli,G. 131 Ghika,M. 86 Ghose,S. 149 Giannopoulou, J. 138 Giardino, D. 142 Gicquel,C. 44 Gieselmann, V. 156 Giglio, S. 40 Gil, A. 102 Gilgenkrantz, S. 116 Ginglinger, E. 123 Giollant,M. 134 Giovanardi Rossi, P. 62 Girardet, A. 62 Girginoudis, P. 99 Giudici,G. 139 Giugliani, R. 91 Gizatullin, R.Z. 61 Glazar,R. 113 Glowaczover, E. 46 Glowaczower, E. 32, 57 Glukhova,L. 144 Goburdhun, J. 108,122 Gobuzov, R. 52 Gobuzova, R. 133 Goguel,A.F. 144 Goizet,C. 124 Golan, R. 48 Goldman, B. 48,55 Gole,L.A. 90 Gomez, E. 31, 57 Gómez-Espí, M. 31 Gomez-Espi, M. 73 Gonçalves, J. 103 Goncharova, E. 97 GonzálezTizón, A. 151 González-Tizón, A.M. 152 Gordon, L.A. 62 Gorman, P. 148 Görög,J.P. 51 Gorovaya, A.I. 69 Goshen, Y. 52 Grace, E. 138 Gracia,R. 155, 157 Gradassi, C. 86 Grammatico, B. 109 Grammatico, P. 106, 109 Granada, I. 138 Granata, P. 143 Grancho, M. 32 Grao,P. 58,96,109 Gras,E. 147 Greene, S.A. 107 Greer, A. 79 Gregoric, J. 83 Griffin, D.K. 26,27,61, 152 Grigori, P. 66 Grigoriadou, E. 86 Grigoriadou, M. 79, 121 Grimoldi, M.G. 131 Grinchuk, NA. 157 Grinchuk, T.M. 70,83,

144, 155 Grishko,V. 157 Grisoli,J.F. 149 Grivina, I. 156 Grkovic, S. 109, 155 Gronwald, J. 51 Graver, L.E. 130 Grubesic, Z. 156 Grün,F. 50 Gruson, N. 56 Gualandri,V. 147 Guanciali Franchi, P. 28 Guanti,G. 103 Guc-Scekic, M. 91,143 Gug,C. 125 Guichaoua, M.R. 48 Guichet,A. 91 Guillemain, C. 48 Guillou,L. 51 Guitart, M. 87, 109 Gül,D. 75, 117 Gülen,O. 118 Guleyuk,N.L. 91 Gumenetzkij, I. 32 Guoqiong, Q. 23 Güran,S. 117 Guran,S. 95 Gurotti,G. 84 Gusina,N.B. 45 Gustavsson, I. 36 Gustavsson, P. 59 Guthauser, B. 84 Gutierrez, S. 30 Gutkowska,A. 110, 128 Guyon, F. 46, 98, 99 Gyftodimou, J. 110, 121 Haan,E. 28 Haan, N.A. de 37 Haar,F.-M. 64 Haas,O,A. 34,59,120, 135 Hadjimarcou, M. 64 Hadjimarcou, M.I. 63, 101, 122 Hadjimarkou, M. 66 Hagemeijer, A. 46, 128 Häggarth,L. 51 Hallman,H. 132 Hamers, A.J.H. 42, 65 Harbott,J. 47,139 Harding, B. 148 Harhalakis, N. 139 Harrington, B. 80 Harrington, J. 79 Harris, P.C. 28 Harrison, C.J. 134 Has, C. 143 Hassan, T. 122 Hatzipouliou, A. 110 Haus, O. 131 Hausmann, M. 56, 64 Havryluk, Y.J. 71 Havrylyuk, Y.J. 156 Hawkins, J.M. 134 Hayes, H. 38,151 Haznedaroglu, R. 136 Heim, S. 144 Heiman, P. 147 Heinonen, K. 132,136 Held,K. 43 Helderman, A. 88 Hellani,A. 58,76 Hemery,B. 32, 143 Henke,B. 107 Henn, T. 120 Henry, E. 107 Herbert, M. 49 Herens, C. 80,99,99, Ill,

132 Hermanne, J.P. 132 Hernandez, J.M. 138 Herranz,R. 31,73 Herrick, J. 33 Heurtevin, P.C. 93 Hindkjær,J. 54 Hinkel,G.K. 63 Hitrec, V. 60,69, 132, 136 Hliscs, R. 32,36 Hnateiko, O.Z. 71 
Hnatejko,O. 127 Hoenigsberg, H.F. 55 Hoffet,M. 89 Holtgreve-Grez, H. 51 Holtgreve-Grez, H.H. 58 Holzgreve, W. 45 Horovitz, J. 46, 98, 99 Horsley, S. 26 Houghton, J.A. 27, 79 Howell,R.T. 35 Huber,Ch. 50 Hübner,H. 70 Huebner,K. 50 Hugues, J.N. 76 Hulejuk, N. 127 Huseyin, S. 29

Ianniruberto, A. 103 Iannuzzi, L. 151 ibrahimoglu, L. 92 Iglesia, C. de la 77 Ignatova, T.N. 83, 157 Ikonen,A. 124 Ilieva,P. 110, 125 Imamoglu, N. 69 Imirzalioglu, N. 117 imirzaliogiu, N. 75 Imresh, S. 61 Incarbone, M. 50 Indrák, K. 132 Insua, A. 153 Intxausti, J.I. 39 Ioannou, I. 64 Ioannou, P. 27, 63 Ion, A. Ill Ion, R. 124 Ischpekova, B. 125 Ivanova, I. 75 Jacky,P.B. Ill Jaffray,J.Y. 104 Jakab,Zs. 141 Jalenques, I. 104 Jamar,M. 80,99, Ill, 132 Jamieson, A. 117 Jamieson, C.R. 110 Janny,L. 82,83 Jans,H. 138 Jariego,C.M. 155, 157 Jarmuz, M. 67, 68 Jarosová, M. 132 Jarvis,A. 148 Jaszczak, J. 154 Jaszczak, K. 151, 154 Jauch,A. 23,51,58,66 Jeison, M. 52,133 Jiang, F. 62, 147

Jimenez, J. 58,96,109 Jimenez, M.D. 109 Jkarkava,N. 80 Jobanputra, V. 78, Ill Joly,G. 83 Jones, L. 47 Jong, B. de 65 Jong, P.J. de 27 Joos, S. 26 Jorge, G. 128 Jotterand Bellomo, M. 129 Jovanovic, A. 61 Jowitt, S. 130 Jungerman, M. 113 Jutzkevich, R. 135

Kadin,M.E. 133 Kajter,P. 136 Kalaitsidaki, M. 123 Kale,G. 87 Kalla,J. 133,137 Kallergis,G. 137 Kallioniemi, O. 23 Kalousek, D.K. 80 Kammoun, M.R. 129 Kanhai, H.H.H. 88 Kaplina,V. 71 Kapovic,M. 72,95 Kara,N. 118 Karabela,M. 112 Karadima,G. 65,79, 110, 123 Karaharju, E. 140 Karakas,P. 38 Karaman,B. 58, 115 Karmanov, M.E. 56 Kashuba,V.I. 61 Kaski,M. 124 Kasuba,V. 70 Kattamis, Ch. 123 Katzenberger, T. 133,137 Kavetsky, R.E. 142,146 Kayserili,H. 58 Kazakov, A.E. 64 Kazantzeva, L.Z. 66, 79, 126 Kchusnutdinova, E. K. 154 Kearney, L. 26,139 Kellie,S. 148 Kelve,M. 61 Kerndrup,G. 133 Keser,i. 114 Kessler,R.G. 91 Keszka,J. 151 Kharchenko, T.V. 73 Khitrikova, L.E. 80,92, 125 Khusnutdinova, E.K. 126 Kienle, D. 23 Kilby,M. 90 Kirchhoff,M. 60 Kirillova, E.A. 112 Kirillova, IA. 92 Kiss,C. 61, 141 Kisseljov, F. 154 Kisseljova, N. 154 Kitsiou-Tzeli, S. 65, 122 , 123 Kivioja,A.H. 140 Kjeldsen, E. 53 Kladny,J. 144

Kihç, G. 58,92 Klein, G. 61 Klestova, Z.S. 70 Klimkina, I.I. 69 Klumper,F. 88 Knight, S.J. 26 Knol,I. 148 Knoll, J.H.M. 133 Knuutila,S. 140 Koch,J. 33,53,59, 138 Kocki,J. 59,133 Koduru, P.R.K. 136 Kokalj-Vokac, N. 59,155 Kokkinou,S. 92 Kolesnik, Ja.F. 146 Kolialexi,A. 94,112 Kolkman,P.H. 88 Kolltner,Y. 52 Kolotii,A.D. 79 Kotowska, J. 67,68,113 Kølvraa,S. 54 Komissarova, E. 154 Kondakova, O. 75 König,M. 59 Kopaliani,N. 80 Korenberg, J.R. 105 Korkach,V. 68 Korniyenko, Y.O. 156 Korolevich, Y. 32 Korzanovskaya, M.V. 29, 127 Kosmaidou, Z. 66,137 Kostrova, L.N. 72 Koszlolanyl, Gy. 136 Kosztolanyi, G. 79 KotlarekHaus, S. 131 Kotzot,D. 103 Kouka,M. 86,112 Koukoulli,R. 101 Koulischer, L. 41,80,99, Ill,132 Kouvaris, I.R. 31 Kovacs, A. 38 Kovalev, C.I. 92 Kovaleva, N.V. 80,92 Kovalyova, Z.V. 70,144 , 155 Kowalczyk, J.R. 59,133 Kozlova, S.I. 56 Kracun, I. 156 Krajewska-Walasek, M. 110,119,128 Krapiva, G.A. 92 Krasevic, M. 95 Kraus,J. 27,55 Kravcenkiené, I. 93 Kravchenko, S. 156,157 Kravetz,B.M. 66, 126 Kravtsov, V.Y. 71 Kravtsov, V.Yu. 69 Krawczyriski, M. 67 Krejci,K. 59 Kristesashvili, J. 80 Krivich, I.P. 112 Kroisel,P.M. 63 Kronberger, G. 120 Kronborg, O. 144 Kroshkina,V. 156 Krumina, A. 156 Krzystolik, Z. 150 Kucheria,K. 78,Ill,113

Cytogenet Cell Genet Vol. 77, No. 1-2, 1997

161

KucheriaK. 149 Kucinskas, V. 93 Kujawski, M. 148 Kulechov, N.P. 76,101 Kuleshov,N. 134 Kuleshov, N.P. 29, 76, 
117,127 Kulkarni, S. Ill, 113 Kumar,H. 149 Kunej,T. 83 Kusina,N.Y. 117 Kuznetsova, T.V. 125 Kuznetzova, T. 33,87,96 Kuzucu, A. 141 Kwiatkowski, F. 32 Kwiatkowski, M. 59

Kyrychenko, N.G. 60 Kyrychenko, V.S. 60

Labadaridis, I. 121 Labar,B. 132,136 Lacerda, A.F. 89 L•acka, K. 67 Lacombe, D. 124 Lakiza, O. 71 Lalchev, S. 71, 125 Lamb,O.A. Ill Lampel, S. 25 Lança, A. 93 Lança,A.P. 89 Lança. A. 104 Landegren, U. 59 Lapierre, J.M. 60 Larizza, L. 50, 52, 126, 142, 145, 147 Lasan, R. 60, 132 Latos-Bieleńska, A. 67, 68, 113 Lauras, B. 101 Lawrence, D. 136 Lawrie, N.M. 26 Lebbar,A. 113 Lebeau, J. 63 Lebiocka, J. 53 LeBouc, Y. 44 LeBourhis, C. 84 Lechat, X. 46 Lederer, G. 122 Lee, B. 57 Lee,C. 152 Leegte, L.K. 65 Leeman, M. 129 Lefort,G. 62,114 Legius, E. 24, 108 Leibundgut, K. 142 Leite,D.P. 96, 119 Leite,R.P. 97 LeLorc'h,M. 84, 114 Lemery, Pr.D. 46 Lemos,R. 96,119,120 Lena, A. 149 Lengauer, C. 58 Leone, L. 88 Leroy, J.G. 66 Lesenfant,S. 99 Lesniewicz, R. 119 Lespinasse, J. 44, 114 Lestou, V.S. 80 Letica, Lj. 60 Leveziel, H. 151 Levin, L.M. 48

Levron, J. 48 Lévy-Mozziconacci, A. 93 Lhomme, A. Ill Li,C. 51 Li, S. 115

Lichter, P. 25, 26, 52, 64 Lichtner, P. 122 Liebaers, I. 100, 119 Ligas,C. 105 Linden, J.C. v.d. 115 Linder,B. 47 Lipsi,M.R. 158 Lishner, M. 127 Lishtvan, L.M. 92 Litmanovitch, T. 127 Little, G. 23 Little, S. 34 Lituania,M. 45, 100 Livescu, B.E. 152 Livi,C. 75 Livolsi, A. 116 Livshits, L. 115, 157 Livshits, L.A. 154, 156 Liyanage, M. 24 Lloveras, E. 131 Logghe, K. 66 Loginova, Yu.A. 71 Lombardo, M. 143 Londvai, G. 136 Loneus, W. 65 López-Piñón, M.J. 153 LopezYarto, A. 129 LoRe,M.L. 105 Los,F.J. 44 Losa,M. 142 Loupal,G. 152 Lowther, G.W. 45 Lozic, B. 105 Lozynska, M.R. 71 Lubetzki, J. Ill Lubinski,J. 144, 150 Lucas, J.L. 97 Luciani, J.M. 48 Lukash, E. 75 Lukash,E.N. 112 Lüleci, G. 114, 115, 141 Lundsteen, C. 60 Lungeanu, A.G. 134 Luño,E. 138 Lushnikova, T. 61 Lyberatou,E. 66, 122, 137 Lygeratou, E. 98 Lysevich, A.N. 75 Maahr,J. 60 Maazoul, F. 129 Macek,M. 93 Macé,B. 83 Macville, M. 24 Madan,K. 95,115 Madgar, I. 48 Magaud,J.P. 130 Maglajlic,S. 109, 155 Maheshwar, M.M. 28 Mahlamäki, E. 132 Mairal,A. 104 Mak,J.W. 52 Makowska, I. 131 Malarchuk, S. 115, 156

Malet,P. 32,64,66,74,

$79,82,104,122,126$,

127, 134, 143 Malfoy,B. 82 Malgara, R. 145, 147 Malinowski, A. 70 Malisheva, LA. 117 Maliyiannis, P. 86 Malkov, S. 61 Malpica, N. 36 Mamaeva,S.E. 139 Manadian, K.K. 64 Manfroi, E. 86 Mann,S.M. 26,61 Manoir, S. du 24 Manola,K. 86 Manouvrier-Hanu, S. 57 Mansour, S. 110 Mantero, M.M. 77 Maral,I. 107 Marcos, R. 30 Mardneau, M. 35 Margarit, E. 89,115 Margaritis, K. 31 Mariani, S. 89 Mariotti,M. 86 Markovic-Glamocak, M.

136 Marín,J. 129 Maro,B. 78

Marques, B. 93, 103, 128 Marques, R. 93, 103 Martens, P.R. 94 Martin, A. 131 Martin, R.H. 81 Martineau, M. 134 Martinelli, B. 86 Martinet, D. 129 Martinez-Pasarell, O. 81 Martin-Pont, B. 76 Martins, L. 55,63 Martin, M.L. 138 Martinez, B. 102, 128 Martínez-Flores, 1.81 MartínezLage, A. 151, 152 Martinez-Pasarell, O. 78, 85 Martos, J. 58,96, 109 Marynen, P. 57 Marzella, R. 34 Mascheretti, S. 90 Maserati, E. 137 Massari, S. 158 Massimo, L. 146 Matsuyama, H. 51 Mattei, M.G. 64 Matteucchi, C. 57 Matthiesen, P. 140 Mavrou,A. 94, 112, 123 Maye,U.C. 130,134 Mayr,B. 152 Mazurczak, T. 53 Mazurek,M. 68, 126 Mazurier, S. 83 Mazza,V. 90 Mazzola, D. 143 McCullagh,P. 47 Mc Elreavey, K. 113 McGiffert,C. 27 Meagher,K.L. Ill Mebes,G. 136 Mecucci, C. 47, 57 Medeira,A. 103, 104, 105 Medeiros, P. 116 Medicina, D. 77 Mehes,G. 135 Mehes,K. 79,136 Meliksetian, M.B. 70, 144, 
155 Mellado,F. 87 Melle,G.van 129 Meller,Y. 52 Melo,P. 104 Meltoranta, R.-L. 132

Menezo,Y. 58,76,82,83,

85 Menzies, A. 45 Mercier, S. 81 Merciuc, N. 116 Merciuc, N. 94 Merino, A. 135 Meroni,E.

131,143 Mertens, S. 100 Mesogitis, S. 137 Metaxotou,C. 94,108,

112,123 Mezzelani, A. 145 Méndez,J. 151, 152,153 Micci,F. 106 Michaelidis, C. 138 Michalet,

X. 28, 33 Michalová, K. 135 Michalski, A. 148 Michelakakis, E. 137 Michelland, S. 61

Midro,A.T. 119 Migliore, L. 116 Migliore, L. 30 Mignon, C. 64 Miguez, L. 94 Mihál,V. 132

Mihatsch, M.J. 51,62, 147 Mihaylets, L.P. 77 Mihov,V. 125 Mikhelson, V.M. 116 Mila,M. 109

Miliuviené, E. 93 Miller,K. 41,43 Minderer, S. 105 Minelli,A. 137 Minelli,E. 143 Mingarelli,

R. 28 Miniou, P. 57 Minoletti,F. 50,52,145 Miny,P. 43 Miozzo,M. 52,89, 145 Mirkova, E. 71

Miró,R. 141,142 Mirò, R. 147 Miró, R. 73 Miro,R. 94 Mironov, P.I. 126 Mitelman, F. 25

Mitsouli-Mentzikof, C.

139 Moch,H. 51,62,147 Moga,M. 125 Monachov, V.V. 64,67 Monakhov, A.S. 145 Moncla, A. 64 Mondello, C. 145

Monreal, S. 135 Montagnac-Bissay, F. 82 Montanari, L. 90 Montbel, I. 74 Monteagudo, L.V. 39

Montserrat, E. 135 Moorman, A.V. 35,134 Mor,C. 52 Morando, A. 146 Mordalska, A. 70

Mordenti,C. 109 Morel,F. 81 Moreno, M. 31,73 Morerio, C. 146 Morescalchi, A. 37,153

Morescalchi, A.M. 37 Morizio, E. 28 Moroz, J. 135 Morris, A. 45 Morris, M.A. 107 Morrison,

N. 117 Mosesso, P. 72, 141 Mossafa,H. 87,97 Mosse, LB. 72 Mota,C. 96,119 Mota,C.R. 104, 105 Motta,S. 39 Motte,J. 56 Mourazos, M.J. 152 Mrad,R. 129 Mrózek, K. 136 Mrsic, M. 136

Mrsic, S. 136 Mühlematter, D. 129 Mühlig, P. 32, 36 Müller, S. 24 Müller-Hermelink, H.K.

133, 137 Murdoch, A. 49 Murken,J. 122 Murnane, J.P. 55 Musienko,S. 156, 157

Müslümanoglu, H. 102,

150 Musso,K. 50 Muzinic, D. 60 Myklebost,O. 140

Nacheva, M. 146 Nakopoulou, L. 138 Nardo,T. 145 Narducci, P.L. 86 Nash,RW. 63 Natarajan, A.T. 72, 141 Naumchik, I. 121 Navarro, J. 77, 82 Navlet,J. 31,57 Nazarenko, S.A. 118 Nemet,

D. 136 Nemtsova, M.V. 29, 117 ,

127 Neronova, E.G. 73 Nesina, I.P. 146 Nesprjadko, S.V. 146 Neuhaus,C. 50 Neumerzhitskaya,

L. 68 Nicolae,I. 152 Nielsen, A. 54 Nieuwint, A.W.M. 95, 115 Niggli,F.K. 142 Nihoul-Fekete,

C. 122

162

Cytogenet Cell Genet Vol. 77, No. 1-2, 1997

Nikiforov, A.M. 69,71,73 Nikiforova, O.K. 112 Nilsson, M. 59 Ning, Y. 24 Nishi,M. 122

Nocera, G. 95 Nogues, C. 38 Nogués,C. 81,85 Nolan, A. 79 North, M.O. 82,84 Nosko,M. 71 Nousiainen, T. 132 Novak, A. 85, 130, 137 Novak, A.Z. 62 Novikov,P.V. 29, 127 Novíkova, I.V. 92 Nunes, J. 104 Nunez, R. 131 Nunez, T. 102 Nutmubidze, N. 90 Nuzzo,F. 145

Obersnel, V. 72 Obersztyn, E. 53 O’Brien, K.O. 50 O'Brien, P. 24 O'Brien, P.C.M. 27, 39, 63, 152 Odero, M.D. 129 Odierna, G. 37 Ogur,G. 117,136 Ogur,G. 75 Ogur,G. 95 Ohl, S. 26 O’Keeffe,C. 84 Ökten, G. 118 Oláh,É. 141 Oldrini,A. 95 Oliva,R. 115 Olivares,P. 31,73 Oliva Teles, N. 74 Oliver, A. 155, 157 Oliver, M. 82 Olmo,E. 153 Olson, C.L. Ill Önderoglu, L.S. 117 Onderoglu, Ls. 95 Önol,B. 87 Orera,M. 31,73 Orlyansky, A. 96 Orlyansky, A.V. 75 Ortiz de Solórzano, C. 36 Oruçkaptan, H. 140 Osipova,G.R. 56 Ostroverkhova, N.V. 118 Ott,G. 133,137 Ott,M.M. 133 Ottavianelli, L. 72, 141 Özbilim,G. 141 Özcan,O. 117 Özdemir,M. 88, 102, 118, 150 Ozdemir, O. 118 Özdemir,Ö. 119, 123 Özen,S. 75,117 Ozkul, Y. 68, 69 Özön,H. 88, 118 Paclovic, S. 109 Padilla-Nash, H. 24 Pages, M.P. 58 
Pagoulatos, G. 65, 123 Pajor,L. 136 Palau,L. 138 Palitti,F. 72, 141 Palka, G. 28

Palmares, C. 96, 119, 120 Pals, G. 95 Pan,Y. 51 Panarello, C. 146 Panasiuk,B. 119 Panattoni, A. 143 Pandelia,E. 110, 121 Pangalos, C. 49, 58, 86, 99,

112 Pantelias, G.E. 31,72, 138,

139 Pantic,M. 130 Pantic,M. 137 Pantic,M. 85 Pantic,M.D. 62 Pantova,I.G. 80,92,125

Papandreou, U. 112 Parada, R. 151 Parenkova, T. 75 Paringaux, C. 91 Parlier,V. 129

Parmeggiani, A. 62 Pásaro, E. 78 Pascual, I. 39 Paskins, L. 34 Pasquali, F. 137 Passarge, E. 107 Passerini, I. 75 Pastorino, U. 50 Pater, J. de 148 Patkin,E.L. 82 Patsalis, P. 66 Patsalis, P.C. 27 , 63, 64,

101,122 Patton,M.A. 110 Pavese, N. 116 Pavlenko, M.A. 70, 144,

155 Pavlovic, S. 155 Pavon,C. 144 Pearson, P. 148 Pecori-Giraldi, F. 142 Pedersen,B. 138

Pedeutour, F. 50 Peixoto,A.L. 119 Pellegrini, S. 75 Pellegrino, V. 77,88 Pellestor, F. 48,53,62,

114 Pendic,V. 85 Pendina,A. 33,96 Pennacchio, A. 88 Perani,P. 39 Percin,E.F. 118 Perçin,E.F. 119,123 Pereira,A. 96, 119, 120 Perez, A. 94 Perez, M.M. 58 Perez-Juana, A. 129 Perfumo, C.

62 Perich,R.M. 87 Perissel, B. 143 Peristeris, P. 138, 139 Pernin, D. 32 Perriot, S. 32 Persani, L. 142 Perucatti, A. 151

Pescia, G. 101 Peterlin, B. 83 Petersen, M. 65, 123 Petersen, M.B. 79, 110,

121 Petit, P. 24, 37, 40 Petrij, F. 124 Petronijevic, D. 91,143 Petropoulos, P. 86 Petrovic, O. 95

Petrozzi, L. 116 Pezzolo, A. 97 Perez, A. 131 Perez, M.M. 78,96, 109,

138 Pfeffer,J. 84 Philip, N. 93 Phillips, O.P. 94 Phocas,I. 98 Pichon, I. 83,85 Pierangeli, C. 86

Pierluigi, M. 62 Pierotti, M.A. 50,52, 145 Pikalová,Z. 132 Pilinskaya, M.A. 73 Pilotti, S. 50,52,

145 Pinto, M. 97 Pinto, M.R. 74 Piombo, G. 77 Piquet, C. 93 Pirc-Danoewinata, H. 120 Pistoia, V. 97 Pitrez,J. 120 Plataniotis, G. 31 Pleskach, N.M. 116 Plochl,E. 120 Plotnikova, S.I. 72

Pluchon,E. 97 Podleshchuk, L.V. 97 Pohlschmidt, M. 45 Polishchuk, L.Z. 146 Politis, G. 138, 139 Polityko,A. 120, 121 Pomerantseva, E.I. 76 Pomili,G. 86 Pommier, J.P. 63 Pompetti, F. 28 Ponsà,M. 153 Ponsa, M. 38 Ponsot, G. 124 Popadic,T. 91, 143 Popescu, C.P. 36 Popowska, E. 110 Portero,J.A. 128 Portugal, A. 97 Poscente, M. 109 Pospísilová, D. 132 Potoczek, S. 131 Poulakidas, E. 139 Powell, R. 27 Powis, S.H. 54 Pozo,F. del 36 Prang, N. 29 Prapa,Z. 137 Preciado,C. 58,96,109 Prentice, H.G. 138 Price, D.J. 74 Príeto,E. 128 Prieto,F. 138 Prieto,M.J. 31,73 Prieur,F. 101

Prokofieva, V.V. 116 Proshin, S.N. 71 Prozorova, M.V. 80,92, 125 Pugovkina, N.A. 83, 157 Puiu,M. 125 Pujol, R. 58,96,109 Pulina,G.A. 76 Puranakanitstha, C. 50

Qumsiyeh,M. 43,76,83, 85

Rabsztyn, A. 154 Rademaker, A. 81 Radice,M.T. 145 Radice, P. 52 Radman, I. 136 Radojcic, Lj. 85 Radojcic Badovinac, A. 68 Raff,R. 28,122 Ragni,N. 45, 100 Ragoussis, J. 54 Ramirez, M.J. 30 Randic, Lj. 68 Rao,K,W. 136 Rapp, M. 74 Ratnam, S.S. 90 Ravine, D. 28 Razumovskiy, V.G. 60 Redi,C. 90 Regan, R. 26 Rego,I. 152 Reichelt,T. 63 Reifenberger, G. 52 Reifinger,M. 152 ReisLima,M. 104,105 Reiss,J.A. Ill Rendeiro,P. 96, 119, 120 Resic,B. 105 Reynaud, M. 104 Rhead,M.M. 74 Ribas,M. 147 Riboni,R. 145 Ricco,A.S. 34 Richter,J. 51,62, 147 Ricoul,M. 63 Ried, T. 24, 34 Righi,R. 143 Rigola,M.A. 73,94 Rinaldi, R. 109 Rio, P. 32 Rios, R. 138

Ristic,S. 95 Rittinger, O. 120 Riva,C. 51 Riva,P. 52, 145, 147 Rives, N. 83 Rizos,D. 98 Rizzi,N. 142 Robert, M. 44 Roberti,M.G. 128 Robert-Nicoud, M. 61 Roberts, E. 90,98 Roberts, K. 138 Robinson, W. 103 Robledo,M. 128 Robson, L. 29 Robson,L.G. 121 Roccella,F. 106 Roccella,M. 109 
Rocchi,M. 26,34 Rocha,E. 129 Rocha, T. 104 Rodewald,A. 134 Rodriguez, M.L. 57 Rodrigues,C. 103 Rodrigues, D. 84 Roeckel, N. 64 Rogel-Gaillard, C. 38 Rogoz, I. 121 Rogoz,S. 121 Roizes, G. 64,67 Rollet,J. 58 Romanini, G. 88 Ronchini, C. 149 Roosmalen, J. v. 88 Rosanda, C. 146 Roschke, A. 24 Rose,N. 27,63 Rosell,J. 115 Rosenberg, C. 52 Rosenwald,A. 133, 137 Rost,I. 122 Rosty,C. 147 Rousseau, O. 89 Rousseau-Merck, M.F. 147 Roy,S. 28 Roy-Doray,B. 123 Roylance, R.R. 148 Rozgaj, R. 70 Rubinstein, J.S. Ill Rubtsov,N.B. 118 Ruiz,B. 99 Rumpler,Y. 82 Rumyantseva, N. 121 Rus,D. 143 Russo, I. 146 Rybalko,A. 93

Sabatier, L. 55,63 Sabatino, G. 28 Saccone, S. 39 Saha,V. 47 Sahai,K. 136 Sahlén,S. 102 Saias,J. 48 Sakarelou,N. 137 Sala,E. 89 Salamalekis, E. 98 Salamanchuk, Z.Y. 71 Salanueva, I. 102 Salek-Zupanclc, S. 136 Sales, M. 107 Salvadore, M. 143 Sambani,C. 138,139 Sampson, J.R. 28 Sánchez-Fayos, JA. 128 Sanchez-Hombre, M.C. 30 Sanoudou, D. 63 San Roman, C. 129 San Roman, C. 30 Sanseverino, M.T. 91 Sans-Sabrafen, J. 131. Santi,F. 97 Santos, A. 30,36 Santos, H. 104,105 Santos, L. 97 Santos, R. 119 Saraiva,J. 104,105 Sarandakou, A. 98 Sard,L. 50,145

Cytogenet Cell Genet Vol. 77, No. 1-2, 1997

163

Sarda,P. 114 Saredi,M. 146 Sarmento, J.C. 120 Sarri,C. 121 Sartorius, G. 51 Satar,M. 124 Satgé,D. 108,122 Saura,R. 46,98,99,124 Sauter,G. 51,62, 147 Savenko, L.A. 92 Scapoli,G.L. 128 Scarpato, R. 116 Schaaf-Lafontaine, N. 132 Schaal, J.-P. 103 Scherer, S.W. 139 Scheres,J. 148 Schiffer, C.A. 136 Schinzel, A. 24, 102 Schinzel, A.A. 103 Schirrer,J. 107 Schlegelberger, B. 33, 140 Schoell,B. 58 Schoos,R. 99 Schreder, S.A. 92 Schröck, E. 24, 34 Schroder, M. 29 Schroder, W. 63 Schubert, R. 28 Schuffenhauer, S. 122 Schüler, H. 122 Schurra,C. 28,33 Schüssler, L. 56 Schwanitz,G. 28, 107, 122 Schwerdtle, R. 50 Schwerin, M. 37 Sciarratta, G.V. 45,100 Seeker-Walker, L.M. 35,

134, 138, 141 Sedneva,I.A. 91 Seely,L.M. 94 Seghezzi, L. 137 Seidel,H. 122 Selva,J. 76,78,84,114 Senger, G. 32 Serra,J. 94 Serrano, V. 153 Seruca,R. 119 Sessa,F. 149 Sezgin, I. 118 Sezgin,i. 119, 123 Sgouras, D. 99 Shaffer, L.G. 57 Shaki,R. 55 Shamgina, L.K. 97 Shandlorenko, S.K. 80, 92 , 125 Shapira, S.K. 57 Sharma,P. 148 Sharonin, V.O. 64,66,67, 126 Shaw, P. 148 Sheer, D. 54, 148 Shlykova,S. 96 Shulman, L.P. 94 Sideris,J. 86 Siebert, R. 133,140 Siffroi,J.P. 84 Siffroy,J.P. 82 Siimes, M.A. 140 Siljak-Yakovlev, S. 54 Silva,M. 103 Simão,L. 93 Simão,L.R. 89 Simi,P. 137 Simon, M.P. 50 Singha,U. 149 Siorenda,A. 92 Sipahier, M. 118 Sirenko,A.G. 127 Sismani,C. 101, 122 Sitar,G. 90 Sivakumaran, T.A. 78, Ill, 149 Skoog,L. 51 Skordis,N. 122 Skuratovski, I. 133 Skvortsova, T.V. 69 Slater, H. 66 Sleegers, E. 28 Slozina,N.M. 73 Smeets,D. 97 Smirnova, I.S. 150 Smirnova, N.V. 116 Smimova, T.D. 139 Smit,E. 46 Smith, A. 29, 121, 148 Soares,M.A. 108 Sofocleous, Ch. 123 Søgaard,M. 138 Sole,F. 58 Soler,A. 89,115 Solé,F. 96, 109, 131, 135, 138 Solinas-Toldo, S. 25,64 Solomko, A. 71 Soloviev, I.V. 64, 66, 67, 79, 106, 126 Sopko,N. 157 Sørensen, A.G. 133 Sorokina, E.A. 83, 157 Sorokine-Durm, I. 56, 64 ,

73 Sostoa, M. 94 Sotnikova, E.N. 29,127 Soubbotina, T. 75 Sousa,A. 93, 103 Sousková, I. 132 Söylemez, F. 140 Sözübir,N. 118 Sozzi,G. 50,52, 145 Spaccini, L. 106 Speleman, F. 66 Spikes, A.S. 57 Sretenovic, M.V. 62 Sretenovic, Z. 67 Ståh1,I. 102 Stańczak, H. 53 Stankiewicz, P. 53 Stark, B. 52, 133 Starkova, E.V. 69,71 Stavljenic-Rukavina, A. 
136 Stavrides, G.S. 122 Stavropoulou, C. 64 Stefanovich, A. 71,84 Stengel-Rutkowski 44 Stephanoyannis, Y. 92 Stevanovic, M. 67, 91 Stevens-Kroef, M.J.P.L. 65 Stéphan,J.L. 101 Stoian,V. 134 Stoicãnescu, D. 125

Stojanova, St. 110 Stoll,C. 116 Stoll,C. 86, 122, 123 Störkel,S. 50 Stoutenbeek, Ph. 148 Straub,B. 84 Strømkjær, H. 54 Stul,M. 57 Stuppia, L. 28 Stylianidou, G. 101 Subbot,S.T. 72 Sucak,G. 136 Sucic,M. 136 Sukhikh,T.R. 153 Sungu,S. 118 Süngü, S. 119,123 Susca,F. 103 Swanton,S. 138, 141 Swillen,A. 108 Syrrou,M. 65, 101, 123 Szabo,I. 125 Szanyi, I. 136 Szeles, A. 61 Szmeja,Z. 148 Szpecht-Potocka, A. 53 Szpiro-Tapia, S. 87,97 Szyfter,K 148 Szyfter,W. 148

Taborelli,M. 99,149 Tachdjian, G. 60 Taçoy, S. 115 Taine,L. 46,98,99,124 Tamain,J.C. 74 Tan,M.B. 115 Tanasie, M. 121 Tandogdu, Ö. 88,102 Tangari, E. 103 Tangi,I. 127 Tanzariello, A. 34 Taramelli,R. 51, 149 Tarkkanen, M. 140 Tarnow-Mordi, W. 107 Tarunina, M.V. 83 Tarunina, T.M. 157 Tasiopoulou, A. 92 Tassin, F. 132 Tavares,P. 96, 119, 120 Taylor, J.W. 110 Tchirkov,A. 32,46,74,

104, 134 Tease, C. 84 Teixeira, F. 103 Tejedor,M.T. 39 Telvi,L. Ill,113,124 Tembe,V. 148 Temocin, A.K. 124 Templado,C. 78,81,85 Tengström, C. 124 Terzoudi, G.I. 31,72 Testa, A. 116 Teyssier, F. 101 Tharapel, A.T. 35,94 Tharapel, S.A. 100 Theodoropoulos, P. 86 Theuil,G. 130 Thompson, P.W. 28 Thonney, F. 101 Tibiletti, M.G. 51,99,149 Tkachova, N.J. 75 Tocco, T. 64 Todoric,B. 85, 130 Toller, M. 118 Tolmie, J. 117 Toncheva,D. 110, 125, 146 Tornielli,S. 50,52,145 Torres, F. 96, 119, 120 Torricelli, F. 75 Tosi, S. 139 Tournilhac, O. 134 Trafalis, D.T.P. 138,139 Travade, P. 134 Treille-Ritouet, D. 130 Tribukait,B. 51 Trowsdale, J. 54 Trubia,M. 51,149 Truillet,M. Ill Tseghi,C. 108 Tsezou, A. 65, 122, 123 Tsita,K. 98 Tsukerman, G.L. 45, 92 Tudegård, L. 102 Tukiainen, E. 140 Tunca,Y. 75, 117 Tunçbilek, E. 86 Turc-Carel, C. 50 Turilova, V.I. 139 Turk,J. 40 Tymofeyeva, N. 31 Tyukacheva, M.V. 71 Tyukatcheva, M.V. 69 Tziropoulou, M. 108, 112 Ugolotti,L. 45,100 Ulas, V.Y. 106 Ulrich,E. 56 Üstün,M.E. 140 Utziel,L. 131 Vaglenov, A. 71 Vagner-Capodano, A.M. 149 Vaiman, M. 38 Vajarova,R. 110, 125 Vale,F. 103 Vallcorba,I. 30, 129,131 Valle,J. 140 Vallespí,T. 138 VanAssche,E. 100,119 Van den Berghe, H. 57 Van Roy, N. 66 Vanurikhina, E. 65,157 Vaquero, J.J. 36 Varela,J.M. 155, 157 Varouta, A. 92 Vazquez-Mazariego, Y. 30 Vechova,N.V. 66,126 Veen, AY. van der 56, 65 Veiga,A. 76,77 Velagaleti, G.V.N. 94 Veldman,T. 24 Velissariou, V. 66,98, 122 Ven, K van der 28 Venti,G. 86 Verdier,R 85 Verlinskaya, D.K. 125 Verloes, A. 99,Ill Vermeersch, J. 24 Vermeesch, J. 40, 57 Veronese, M.L. 50 Verrelle,P. 32,74 Verschraegen-Spae, M.R. 66 Vessalas, G. 138 Vianello, M.G. 45,100 Vianna Morgante, A. 57 Vicens, E. 81 Victorova, T.V. 154 Vidal,F. 48,85,153 Vidovic,A. 137 Viegas-Péquignot, E. 57 Vieira,L. 128 Viersbach,R. 107 Vig,B.K. 149 Viggiano, L. 34 Viktorov, V.V. 126 Viktorova, T.V. 126 Villa, N. 89,126 Villalón,C. 30 Virolainen, M. 140 Vissers, J. 148 Vlastelic, I. 68 Vögtel,D. 122 Voisin,P. 56,64,73 Volodina,N. 33 Volonté, M. 145 Volontè, M. 147 Volpi,E.V. 54 Volpi,L. 52 Vorobjova, L.I. 142 Vorobtsova, I. 31 Vorsanova, S. 27 Vorsanova, S.G. 64, 66, 67,79, 106,126 Voullaire, L. 66 Vrouvas, J. 31 Vugt, J.M.G. van 95,115 Vujic, M. 67 Vujosevic, M. 61

Wagner, A. 63 Wallace, W.H.B. 138 Warmer, A. 152 Wang,N. 51 Wasko,B. 59,133 Watson, N. 148 Watson, N.W. 121 Wauters, J.-P. 51 Webb, A.L. 49, 85 Weber, R.G. 52 Weber-Matthiesen, K. 140 Weeks, F.F. Ill Weidt,E. 50 Weissenberg, R. 48 Wen,Z.Q. 46,98,99,124 Werner,W. 67 
Wernlund, A.-K. 102 Wertelecki, W. 115 White, I. 102 Whittington, R. 74 Whittle, M. 90,98 Wieczorek, D. 107 Wiegant,J. 52,60 Wienberg, J. 24, 27, 39 Wijmenga,C. 148 Willatt,L.R. 63 Winberg, G. 61 Wisniewska, M. 113 Wisniewska, M. 126 Wiss, J. 60 Wlodarska, I. 46, 57 Woessner,S. 131, 135, 138

164

Cytogenet Cell Genet Vol. 77, No. 1-2,1997

Wojda,A. 67,68 Wofczyท́ski, S. 119 Wolf,D. 56

Wolstenholme, J. 49,85 Wong,Y.C. 90

Yakovlev, A.F. 71 Yakovleva, T.K. 150 Yang,F. 24,39,63,152 Yaroshevich, E.Yu. 97

Yaroslavskaya, S. 65,157 Yartseva, N. 154

Yatsenko,A. 29, 127 Yavuz, G. 136 Yavuz,N. 102, 118,150 Yavuz, Ö. 118 Yerle,M. 37

Yevsikov, A. 71 Yoshihiro, S. 51 Yoshitomi, M.J. Ill Young, B.D. 47 Young, J. 45 Yürksel,A. 92 Yurov, Y. 27

Yurov Yu.B. 64, 66, 67, 79, 106, 126

Zabarovsky, E.R. 61 Zahed,L. 127 Zaizov, R. 52, 133 Zajaczek,S. 144,150 Zakhariev, V.M. 61 Zaklik,S. 52 Zalen-Sprock, R.M. v. 95 Zaletayev, D.V. 29, 117, 127

Zamani, A. 106 Zambela, M. 63 Zattara-Cannoni, H. 149 Zavadil,J. 135 Zavoreo, I. 156

Zawada,M. 67,68, 113 Zawadzka, M. 154 Zemanová, Z. 135 Zera,C. 122 Zerova, T.E. 77

Zerres, K. 122 Zghal,M. 129

Zhang, Y. 140 Zharicova, L.V. 75 Zijlstra,C. 37 Zink,D. 23 Zivanovic, P. 156 Zorn,B. 83

Zosi,P. 121 Zuffardi,O. 40 Zurbitskaja, B. 154

Cytogenet Cell Genet Vol. 77, No. 1-2, 1997

165 